\section{Communication Constraints for Decentralized Stabilizability With Time-Invariant Policies}

\author{
Serdar Yüksel and Tamer Başar
}

\begin{abstract}
In this note, we study communication requirements for decentralized control schemes when sensors and controllers are connected via noiseless digital channels and system noises are bounded. Under various information structures, and within the class of time-invariant encoding and control policies, we quantify achievable rates, and obtain fixed-rate and delayless encoders and decoders that achieve boundedness in the state estimation errors at the controllers. We use distributed coding theoretic approaches to show that when the sensors do not collaborate, it is sufficient for the controllers to share information amongst themselves but not with the sensors. Sensor collaboration, however, leads to improvement in rate performance.
\end{abstract}

Index Terms-Control under communication constraints, decentralized control, distributed coding, multi-agent systems, quantization.

\section{INTRODUCTION}

With the recent advances in the communications technology, the use of digital and wireless channels such as the Internet or bus lines [as in a Controller Area Network (CAN)] in control systems have become common place. Some examples include vehicle systems, and aerospace applications [1], [2] as well as formation control [3]. Further, sensor network applications increasingly involve control systems. In such remote control problems, one major concern is the characterization of the amount of information transfer needed to guarantee a desirable performance. This information transfer would be between various components of the networked control system, as depicted in Fig. 1. One problem of interest is regarding the ability of the controllers to track the plant state under communication constraints through various correlated observations.

In this note, we study such a problem, that is the problem of information transmission leading to stabilizability, under a number of decentralized information structures. In all, we assume the coding and control policies to be time-invariant and of the static feedback type, and that there exists a centralized, or a locally centralized controller. A precise problem formulation is now given next, followed by a brief review of relevant literature.

\section{A. Problem Formulation}

Consider an $n$-dimensional discrete-time noisy LTI system with an $n$-dimensional control input, where each control component has a direct effect on a corresponding scalar state. More precisely, we have the state dynamics

$$
x_{t+1}=A x_{t}+B u_{t}+w_{t}, t \geq 0
$$

Manuscript received May 4, 2005; revised October 16, 2006. Recommended by Associate Editor J. Hespanha. This research was supported in part by the NSF Grant CCR 00-85917 ITR. An earlier version of this paper was presented at the 42nd IEEE Conference on Decision and Control (CDC), Maui, HI, December 2003, and appeared in an abridged form in the conference proceedings.

S. Yüksel was with the University of Illinois, Urbana-Champaign, IL 61801 USA. He is now with Yale University, New Haven, CT 06511 USA (e-mail: serdar.yuksel@yale.edu).

T. Başar is with the Coordinated Science Laboratory, University of Illinois, Urbana, IL 61801-2307 USA (e-mail: tbasar@ decision.csl.uiuc.edu).

Digital Object Identifier 10.1109/TAC.2007.899085

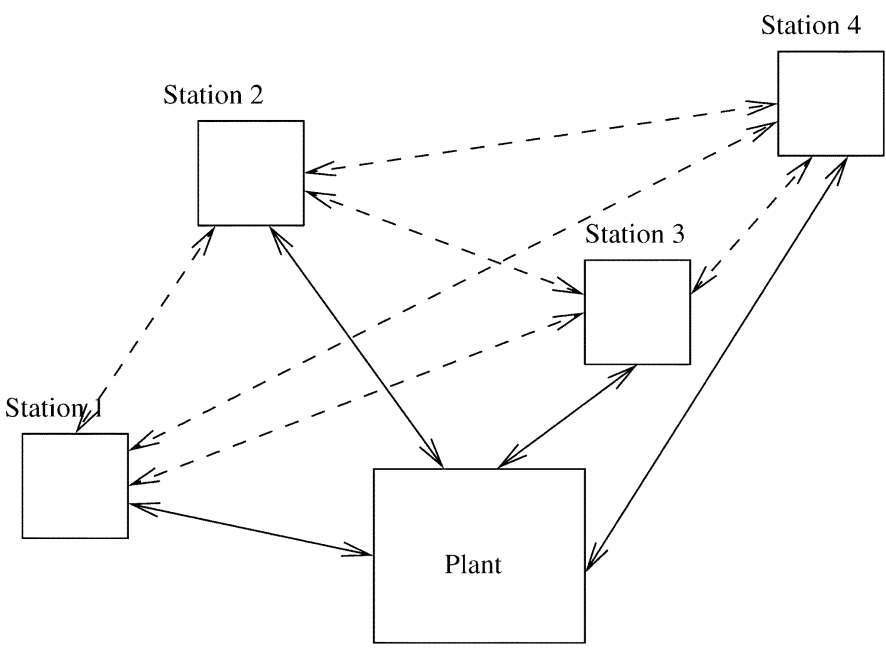

Fig. 1. Decentralized networked control system. Solid lines indicate the interaction between the control stations and the plant. Dashed lines depict the possible communication links between the stations.

where $A$ is an $n \times n$ matrix, $B=\operatorname{diag}\left(b_{1}, \ldots, b_{n}\right)$ is nonsingular, $x_{t}$ is the state, $u_{t}$ is the control, the initial state $x_{0}$ is a continuous random vector with a known distribution with finite support, and $w_{t}$ is an $n$-dimensional zero-mean i.i.d. noise process whose distribution also has bounded support. Each control component (say, $u^{i}$, the $i$ th subcontroller) has access only to the corresponding component of the state $\left(x^{i}\right)$, but with this information transmitted from a sensor to the (sub)controller over a bandlimited noiseless channel.

We denote the encoder output corresponding to $x_{t}^{i}$, transmitted from the $i$ th sensor to the $i$ th controller at time $t$ by $z_{t}^{i}$. We note that given a partitioning of $\mathbb{R}, \mathcal{P}=\left\{\mathcal{B}_{j}\right\}$, where the $\mathcal{B}_{j}$ 's are the subintervals, or bins, with $\mathcal{B}_{j}=\left[\delta_{j}, \delta_{j+1}\right)$, a quantizer $Q$ is a mapping from $\mathbb{R}$ to $\mathcal{P}$. For each $x^{i} \in \mathbb{R}$, we can also identify $Q\left(x^{i}\right)$ with a point $q_{j} \in \mathcal{B}_{j}$, for some $j$ such that $x^{i} \in \mathcal{B}_{j}$. We denote by $\hat{x}^{i}$ the estimate at the controller for $x^{i}$. In addition to allowing each (sub)controller $i$ to have access to $z_{t}^{i}$ at time $t$, we will also allow the controllers to exchange some past information, as to be delineated below. We will identify six information structures (ISs) (see Fig. 2), depending on what is available to and exchanged by the sensors and the controllers. In each case, $I_{r, \text { sensor }, t}^{i}$ (respectively, $I_{\text {, controller }, t}^{i}$ ) will denote the information available to sensor $i$ (respectively, controller $i$ ) at time $t$.

1) Centralized IS: This is the classical case. The sensors and the controllers collaborate and share all the information they have. This case is included here for the sake of completeness, where both the sensors and the controllers can be viewed as single blocks

$$
\begin{aligned}
I_{A, \text { sensor }, t}^{i} & =I_{A, \text { sensor }, t}=\left\{u_{[0, t-1]}, z_{[0, t-1]}, x_{[0, t]}\right\} \\
I_{A, \text { controller }, t}^{i} & =I_{A, \text { controller }, t}=\left\{u_{[0, t-1]}, z_{[0, t]}, \hat{x}_{[0, t-1]}\right\} .
\end{aligned}
$$

2) Decentralized IS Where Controllers Communicate, and Relay Their Estimates to the Sensors:

$$
\begin{aligned}
I_{B, \text { sensor }, t}^{i} & =\left\{u_{t-1}^{i}, \hat{x}_{t-1}, x_{t}^{i}\right\} \\
I_{B, \text { controller }, t}^{i} & =\left\{u_{[0, t-1]}^{i}, z_{[0, t]}^{i}, \hat{x}_{t-1}\right\} .
\end{aligned}
$$

Here the sensors have access to the estimates formed at the control stations as well as the actions taken, but have access to the current value of only their own corresponding states. The controllers share the past estimate and action information, but receive the current coded state information only from their corresponding sensors. The controllers apply 


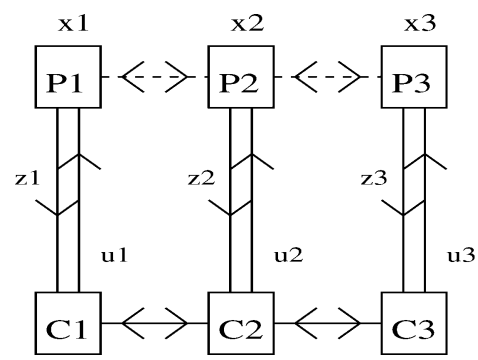

Fig. 2. In IS A, the sensors and controllers have full cooperation, and perform joint encoding and decoding. In IS B, the sensors talk to each other and share what they had sent at the previous time stage, and the control values they had received, but the encoding and the decoding are not joint operations. In IS C, the sensors do not talk to each other and have no access to controller estimates. In IS D and IS E, the controllers collaborate while decoding the state, with the sensors receiving feedback in IS E. In IS F, the controllers communicate with delay.

their control upon the arrival of $z_{t}^{i}$, that is, the data sharing between the controllers takes place after the controllers generate their control values. Here, the decoding at the controllers is not a joint operation.

3) Decentralized IS Where Only the Controllers Communicate, With No Relaying to the Sensors: Here the controllers have the same information as in IS B above, whereas each sensor $i$ has access to the input and output connected to the $i$ th estimator, which is the relevant part of the $i$ th controller. As in IS B, the decoding at the controllers is not a joint operation.

$$
\begin{aligned}
I_{C, \text { sensor }, t}^{i} & =\left\{u_{t-1}^{i}, x_{t}^{i}\right\} \\
I_{C, \text { controller }, t}^{i} & =\left\{u_{[0, t-1]}^{i}, z_{[0, t]}^{i}, \hat{x}_{t-1}\right\}
\end{aligned}
$$

4) Decentralized IS Where Controllers Collaborate, and Relay Their Estimates to the Sensors: Here the sensors have access to the estimates formed at the control stations as well as the actions taken, but have access to the current value of only their own corresponding states. Controllers collaborate while decoding the data, that is, controller $i$ uses the entire $z$ to decode the signal

$$
\begin{aligned}
I_{D, \text { sensor }, t}^{i} & =\left\{u_{t-1}^{i}, \hat{x}_{t-1}, x_{t}^{i}\right\} \\
I_{D, \text { controller }, t}^{i} & =\left\{u_{[0, t-1]}^{i}, z_{[0, t]}, \hat{x}_{t-1}\right\}
\end{aligned}
$$

5) Decentralized IS Where Controllers Collaborate With No Relaying to the Sensors: Here the sensors have access only to the current value of only their corresponding states and what they have sent to the corresponding controller. Controllers collaborate while decoding the data

$$
\begin{aligned}
I_{E, \text { sensor }, t}^{i} & =\left\{u_{t-1}, x_{t}^{i}\right\} \\
I_{E, \text { controller }, t}^{i} & =\left\{u_{[0, t-1]}^{i}, z_{[0, t]}, \hat{x}_{t-1}\right\} .
\end{aligned}
$$

6) Decentralized One-Step Delay IS With Relaying to the Sensors: This is similar to IS B except that the controllers and the sensors exchange information with a delay of one time step

$$
\begin{aligned}
I_{F, \text { sensor }, t}^{i} & =\left\{u_{[t-2, t-1]}, \hat{x}_{[t-2, t-1]}, x_{t}^{i}\right\} \\
I_{F, \text { controller }, t}^{i} & =\left\{u_{[0, t-1]}, z_{[0, t]}^{i}, \hat{x}_{t-1}^{i}, \hat{x}_{t-2}\right\} .
\end{aligned}
$$

Remark: There are two types of side-information in the above structures. One is with regard to the controller estimates relayed to the sen- sors, and the other is with regard to the joint encoding operation at the sensors. We will see that there is no loss due to the absence of the relayed estimate side information, whereas there is loss due to the absence of joint encoding at the sensors.

Throughout, we have $z_{t}^{i}=\gamma_{t}^{i}\left(I_{\cdot, \text { sensor, } t}^{i}\right)$ and $\hat{x}_{t}^{i}=\beta_{t}^{i}\left(I_{\cdot, \text { controller }, t}^{i}\right)$ for some sensor functions (encoders) $\gamma_{t}^{i}$ and estimator functions (decoders) $\beta_{t}^{i}$, where both of these functions will have to be determined. Let $e_{t}^{i}:=x_{t}^{i}-\hat{x}_{t}^{i}$, and $K^{i}$ be the number of levels in the fixed-rate quantizer used by sensor $i$, $1 \leq i \leq n$. Note that then the rate on the channel connecting sensor $i$ to (sub)controller $i$ (say, channel $i$ ) is $R^{i}=\log _{2}\left(K^{i}\right)$, and the total rate for the $n$ channels is $R=\sum_{i} R^{i}$. For two vectors $u, v$, let the notation $u<v$ denote componentwise inequality, i.e., $u^{i}<v^{i}, \forall i$. Finally, let $W\left(e_{t}^{i}\right)$ denote the support width of the random variable $e_{t}^{i}$, that is the width of the connected domain over which all mass associated with the distribution of $e_{t}^{i}$ is concentrated. Then, the main problem we address in this paper is finding a set of achievable total rates over $n$ channels under which the support width of $e_{t}^{i}$ remains finite for all time $t$, and for all $i$. Since the system is controllable, the boundedness in the estimation error implies the existence of control policies which keep the state bounded, and hence, in essence we would be studying here a (decentralized) state-estimation problem. More precisely, we have the following criterion.

Definition: Decentralized Stabilization: For a given information structure (IS), a sum rate $R$ is stabilizing if there exist an $n$-tuple of rates $\left(R^{1}, \ldots, R^{n}\right)$ and corresponding controls $\left(u_{[0, \infty)}^{1}, \ldots, u_{[0, \infty)}^{n}\right)$, compatible with the IS, such that $\sum_{i=1}^{n} R^{i}=R$, and the closed-loop system is stable almost surely (a.s.), that is there exists an $M<\infty$ such that $\lim \sup _{T \rightarrow \infty}\left\|x_{T}\right\|_{\infty}<M$ a.s., where $\|\cdot\|_{\infty}$ is the sup norm.

\section{B. Literature Review and Preliminaries}

Decentralized stabilization has attracted considerable interest in the literature with earlier works including [4], [5], and [6]. With regards to communication theoretic issues, a diverse range of structures and models in this framework have been analyzed by various authors; see, for example [8]-[10] and [11]. Among these, [9] primarily studies the case where the modes observable by the sensors are decoupled. Reference [10] shows that the minimum rate required for stability of multisensor systems with a centralized controller, is the same as the rate required in the centralized case. The analysis in [10] builds on the observation that each of the sensors observes the eigenvalues of the underlying system, with all covered, since the overall system is observable. Reference [13] further studies the multi-sensor problem with constructions. A parallel work is [14], which considers multicontroller systems with time-varying policies and quantifies the minimum achievable rate for any connectivity and graph structure leading to decentralized stabilizability. However, the policies adopted in [14] require time-varying coding and control policies which may be somewhat difficult to implement. Here, we consider instead time-invariant, fixed-rate, state-feedback control and encoding policies, and obtain achievable (though somewhat higher) rates in this context.

Another contribution of this note is to the sensor network literature where various distributed source coding and side information based schemes have been studied; see, for instance [16], [17] and the references therein. We investigate here further a two-fold side information interpretation, one of which is due to the linear evolution of the dynamics, and the other one due to the well-studied distributed source coding nature of the problem. Furthermore, we note that unlike the studies in a strict communication framework, the encoding in a realtime control system has to be not only causal, but also delay-free. We use binning schemes, but because of the zero-delay restriction it is not 
possible to use asymptotic binning arguments that are typically employed in Wyner-Ziv coding [18].

In the balance of this note, we provide in Section II solutions corresponding to each of the six information structures introduced. Section III includes concluding remarks.

\section{Stabilizing RATES}

\section{A. Information Structure A}

As mentioned earlier, in the centralized case the system can be viewed as a single multi-dimensional sensor and same dimensional controller, and hence without any loss of generality the system matrix can be considered to be in Jordan form (with $B$ not necessarily diagonal). This observation leads to a minimum achievable rate that can be obtained from that for each subsystem, as captured in the following result. A proof of this result can be found in [19].

Theorem 2.1: Consider system (1) in a centralized setting. The minimum achievable rate under the stability criterion introduced is $R=$ $\sum_{i} \log _{2}\left(C\left(\left|\lambda_{i}\right|\right)\right)$, where $C(x)$ is the smallest integer strictly larger than $x$.

\section{B. Information Structure B}

In this scheme, the estimated states as well as the control signals are shared between the controllers. Furthermore, the encoder at the sensor has access to the corresponding receiver's information. Since our goal here is to obtain boundedness, innovations will be uniformly quantized where quantizer reconstruction values are taken as the mid-points of the bins. Let us consider the first component: If we subtract the predicted value

$$
a_{11} \hat{x}_{t}^{1}+a_{12} \hat{x}_{t}^{2}+\ldots+a_{1 n} \hat{x}_{t}^{n}+b_{1} u_{t}^{1}
$$

from the actual state $x_{t+1}$, the innovation to be sent will then be an appropriately weighted sum of the individual quantization errors. This observation then leads to the result given below, with the quantizers fixed to be time invariant, and using fixed length codewords. We first introduce some notation: Let $A^{+}$be the matrix obtained by replacing all entries of a matrix $A$ with their absolute values. Let $\mathcal{K}$ denote the class of diagonal $n \times n$ matrices $K=\operatorname{diag}\left(k_{1}, \ldots, k_{n}\right)$, where $(1) /\left(k_{i}\right)$ are positive integers, and further that $K A^{+}$is stable.

Theorem 2.2: For IS B, under the restrictions of time-invariant quantization and fixed length coding, the rate given by the solution of the following optimization problem is stabilizing:

$$
R^{*}=\min _{\mathcal{K}} \sum_{i} \log _{2}\left(1 / k_{i}\right)
$$

Proof: The innovation process is given by $e_{t+1}^{i}=a_{i 1}\left(x_{t}^{1}-\hat{x}_{t}^{1}\right)+$ $\ldots+a_{i n}\left(x_{t}^{n}-\hat{x}_{t}^{n}\right)+w_{t}^{i} \equiv a_{i 1} e_{t}^{1}+\ldots+a_{\text {in }} e_{t}^{n}+w_{t}^{i}$. Let the width of the uncertainty in the quantization error, $e_{t}^{i}$, be denoted by $\Delta_{t}^{i}$. Then the uncertainty interval for $e_{t+1}^{i}$ satisfies

$$
W\left(e_{t+1}^{i}\right) \leq\left|a_{i 1}\right| \Delta_{t}^{1}+\ldots+\left|a_{\text {in }}\right| \Delta_{t}^{n}+D^{i}
$$

where $D^{i}$ is the length of the connected domain (interval) over which the $i$-th noise term has full support. Now using a $K^{i}$-level uniform quantization reduces this by the same factor. Hence, we have the recursion

$$
\Delta_{t+1}^{i} \leq\left(\left|a_{i 1}\right| \Delta_{t}^{1}+\ldots+\left|a_{\text {in }}\right| \Delta_{t}^{n}+D^{i}\right) / K^{i}, \quad 1 \leq i \leq n
$$

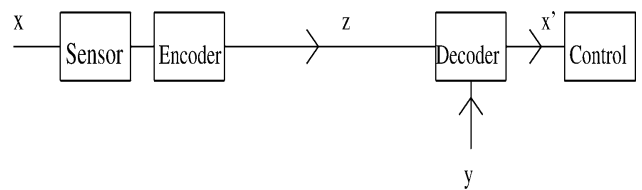

Fig. 3. Wyner-Ziv framework of source coding with side information at the decoder. In our setup $y$ denotes the information shared between the controllers, $z$ is the quantization output at time $t, x^{\prime}$ is the estimate of the state as a function of the side information $y$ and the received data $z$.

and in vector form

$$
\Delta_{t+1} \leq K\left(A^{+} \Delta_{t}+D\right),
$$

where $K=\operatorname{diag}\left(k_{1}, \ldots, k_{n}\right), k_{i}=1 / K^{i}$. The sequence $\left\{\Delta_{t}\right\}$, being generated by a discrete-time LTI system, is bounded (since the initial state $x_{0}$ has bounded support) if and only if $K A^{+}$is stable, that is $K \in \mathcal{K}$, and this corresponds to a total rate of $\sum_{i} \log _{2}\left(K^{i}\right)$. The result then follows.

Remark: In case no information is transmitted for a particular subcomponent of the controller, the number of levels required can be taken to be 1 , since the rate required will be zero for that channel. Hence, the matrix $K$ has elements at most equal to 1 on the diagonal. Furthermore, since $\sum_{i} \log _{2}\left(\frac{1}{k_{i}}\right)=\log _{2} \prod \frac{1}{k_{i}}$, and logarithm is a monotonic function, and $K$ is diagonal, the optimization problem reduces to the maximization problem: $\max \mathcal{K} \operatorname{det}(K)$.

Proposition 2.1: If $A$ is diagonal, the solution of (2) is $R^{*}=\sum_{i} \log _{2}\left(C\left(\left|\lambda_{i}\right|\right)\right)$, where $C(x)$, as defined earlier, is the modified ceiling function.

Proof: When $A$ is diagonal, the condition for stability is: $k_{i}\left|\lambda_{i}\right|<$ 1. If $\lambda_{i}$ is not an integer, we have $(1) /\left(k_{i}\right)=\left\lceil\left(\left|\lambda_{i}\right|\right\rceil\right)$, whereas if $\lambda_{i}$ is an integer, $(1) /\left(k_{i}\right)=\left|\lambda_{i}\right|+1$. Thus, the optimum rate is: $R^{*}=$ $\sum_{i} \log _{2}\left(C\left(\left|\lambda_{i}\right|\right)\right)$.

Proposition 2.2: The optimization problem (2) admits a solution for any matrix $A$.

Proof: Let $\lambda_{i}^{+}, \quad 1 \leq i \leq n$, be the eigenvalues of $A^{+}$. Let $k$ be given by

$$
(1 / k)=C\left(\max _{i}\left|\lambda_{i}^{+}\right|\right)=: C\left(\left|\lambda_{m}^{+}\right|\right) .
$$

Then $K:=k I_{n \times n}$ will lead to a stable $K A^{+}$matrix. The rate in this case becomes $R=n \log _{2}\left(C\left(\left|\lambda_{m}^{+}\right|\right)\right)$. There are only finitely many choices for the matrix $K$ which could be better than the one proposed, and hence there exists an optimal solution.

\section{Information Structure $C$}

For the third structure, where the sensors do not communicate with each other, whereas the controllers share their data and their actions, we have the following result.

Theorem 2.3: Under the restrictions of time-invariant quantization and fixed length coding, the rate given by the solution of the problem introduced in Theorem 2.2 is also stabilizing under IS C.

Proof: Side information, which is the other controllers' estimates, is only available at the controllers (see Fig. 3). We use binning (see [16], [17]) for construction. The approach exploits the assumption that the noise processes are bounded.

The data to be sent, $x_{t+1}^{i}$, can be written as $x_{t+1}^{i}=a_{i} \hat{x}_{t}+a_{i} e_{t}+w_{t}^{i}$, where $\hat{x}_{t}$ is the vector of the state estimates available at each subcontroller, $e_{t}$ is the vector of quantization errors at time $t$, and $a_{i}$ is the $i$ th row of matrix $A^{+}$. Let $W\left(e_{t}^{i}\right)$ denote the support width of the uncertainty $e_{t}^{i}=x_{t}^{i}-\hat{x}_{t}^{i}$, and suppose that $\Delta_{q, t}^{i}$ is a width of the total 


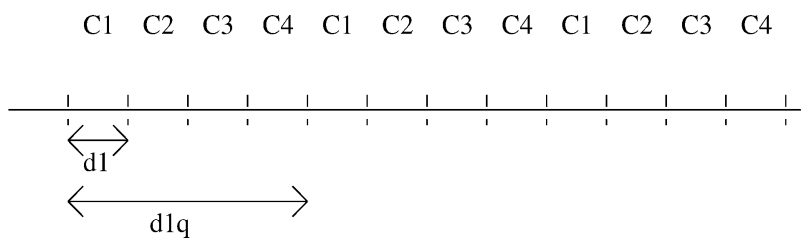

Fig. 4. Binning Structure, the encoder only sends one of the cosets. In our scheme, d1q stands for $\Delta_{q, t}^{i}$ and $d 1$ for $\Delta_{t}^{i}$. The decoder, upon receiving the coset symbol, generates its estimate by finding the bin with the same coset which is closest to its estimate.

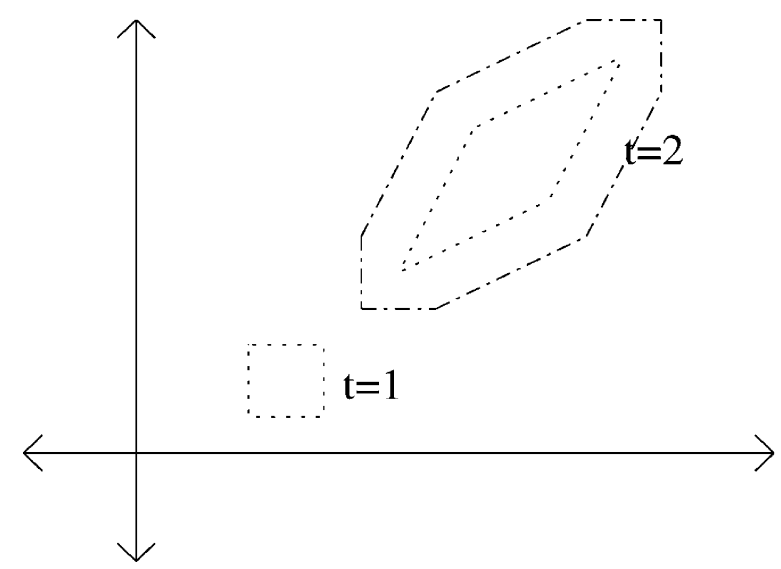

Fig. 5. The evolution of the uncertainty. Note that the evolution is parallel to the directions of the eigenvectors, in addition to the bounded noise effects. The encoders thus send correlated data and can exploit this dependency.

of cosets, as in Fig. 4. Further, let $\Delta_{t}^{i}$ be a quantizer parameter with $W\left(e_{t}^{i}\right) \leq \Delta_{t}^{i}$.

In this scheme, the sensor only informs the receiver as to which coset the state belongs to. The receiver will use the coset and decode the signal using the available side information. The rate required will then be $\log _{2}\left(\Delta_{q, t}^{i} / \Delta_{t}^{i}\right)$. Suppose that the encoder sent $C 1$. In this case, the receiver will need to choose between the possible bins in the coset. There exists a nearest bin without ambiguity, if the condition $a_{i} \Delta_{t}^{i}+$ $D^{i}<\Delta_{q, t+1}^{i}$ is satisfied. If $K^{i}$ levels are used for quantization, then $\left(1 / K^{i}\right) \Delta_{q, t}^{i}$ is a bound on the uncertainty interval at the next stage, which is to be less than $M$. To be able to decode the correct bin using the coset information, we need to satisfy

$$
A^{+}\left(K \Delta_{q, t}\right)+D^{i}<\Delta_{q, t+1}, \forall t
$$

Let $\epsilon>0$, and $1_{n}$ be the vector of ones. For stability it suffices to have

$$
\Delta_{q, t+1}-\epsilon 1_{n} \leq A^{+}\left(K \Delta_{q, t}\right)+D^{i}, \forall t .
$$

The system will be stable if $A^{+} K$ is stable, which is identical to $K A^{+}$ being stable, since for two square matrices $A, B$, the spectra of $A B$ and $B A$ are identical. Define $K$ as the diagonal matrix consisting of the reciprocals of the number of quantization levels on its diagonals. Recognizing this expression from the previous section, an optimization problem can be formulated as in (2).

\section{Information Structure D}

This is the IS where controllers use the entire received data to generate their decoding output. Here the controllers have some room to improve their estimates using the correlated data received by the other

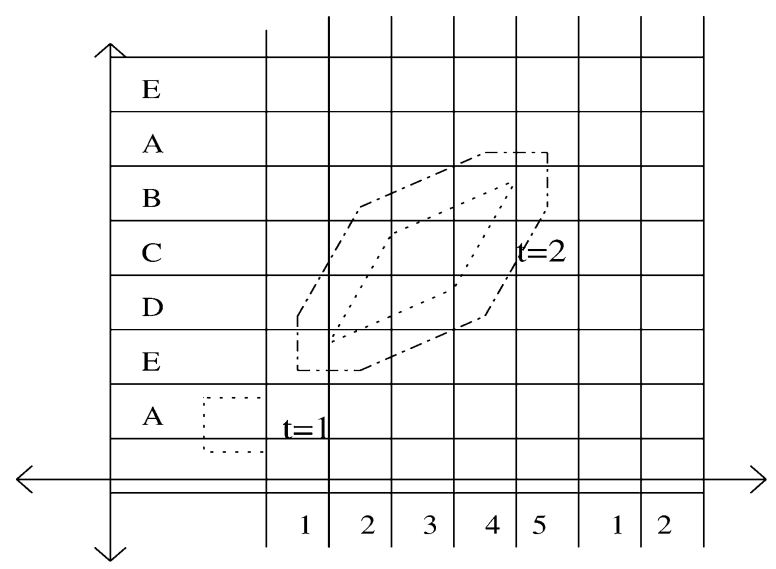

Fig. 6. The evolution of the uncertainty, with the same matrix in Fig. 5 being used. Here, even though the sensors do not know what the controller esimates are, still it suffices to send the coset information. If the controllers do not collaborate then each of their corresponding sensors will send information for five symbols.

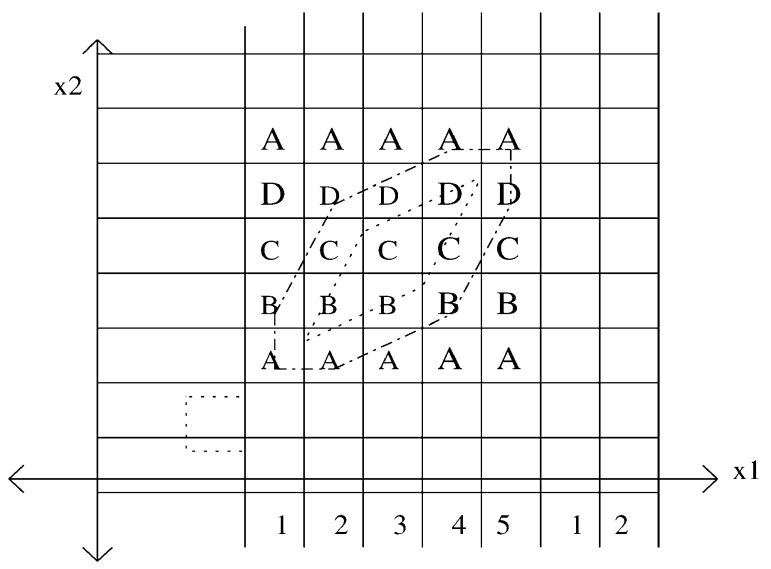

Fig. 7. For any level that sensor 1 has to send, there are only four levels, and not five, that sensor 2 needs to send. Note that each sensor still sends the same coset value for each marginal data. We encode $\log _{2}(25)$ bits if we do not let controllers cooperate. If only the controllers cooperate, we would need $\log _{2}(20)$ bits to be sent.

controllers. At each stage the sensors have access to the uncertainty polygon that they are transmitting (see Fig. 5-8). Thus, each sensor is capable of considering the support sizes of the errors at all the controllers.

Let $V\left(\hat{x}_{t}\right)=\left\{x_{v, t}\right\}$ denote the vertices of the unit cube scaled by $M<\infty$, and centered at $\hat{x}_{t}$. Since the system is linear, one can obtain the following bound on the support size:

$$
W\left(\left(A x^{i}\right)_{t}-A\left(\hat{x}_{t}\right)^{i}\right) \leq \max _{x \in V\left(\hat{x}_{t}\right)} a_{i} x-\min _{x \in V\left(\hat{x}_{t}\right)} a_{i} x+D^{i}
$$

In IS B, this expression was upper bounded, without considering the correlation with the other components of $e$. However, since we allow the controllers to cooperate in this case, there might be cases for which the encoders might send information that takes into account this cooperation at the controllers. Let us introduce

$$
\begin{aligned}
& S_{D}^{i}\left(\hat{x}_{t}\right):=\left\{x_{t}:\left|x_{t}^{j}-\hat{x}_{t}^{j}\right| \leq M, \forall j\right. \\
& \left.W\left(\left(A x_{t}\right)^{k}-\left(A \hat{x}_{t}\right)^{k}\right)<M, k \neq i\right\}
\end{aligned}
$$




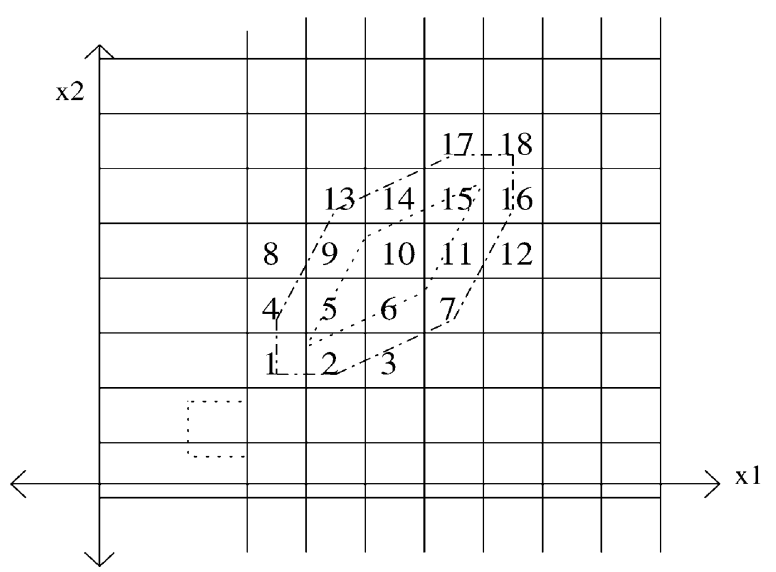

Fig. 8. Joint encoding outperforms independent encoding. If the sensors are allowed to collaborate, there would only be $\log _{2}(18)$ bits to be sent.

and define the number of levels needed to identify the state within a precision of $M$, given the other sensor readings, as

$$
K_{-i}^{i}:=\min \left\{k: k M>\sup _{x_{t} \in S_{D}^{i}\left(\hat{x}_{t}\right)} W\left(\left(A x_{t}\right)^{i}-\left(A \hat{x}_{t}\right)^{i}\right)\right\}
$$

Such a time-invariant $K_{-i}^{i}$ exists because of the finiteness of the decision sets.

To avoid numerical issues in the construction, we provide the following sequential scheme. Define

$$
\begin{aligned}
S_{s, D}^{i}\left(\hat{x}_{t}\right):= & \left\{x_{t}:\left|x_{t}^{j}-\hat{x}_{t}^{j}\right| \leq M, \forall j\right. \\
& \left.W\left(\left(A x_{t}\right)^{k}-\left(A \hat{x}_{t}\right)^{k}\right)<M, k \leq i-1,\right\}
\end{aligned}
$$

and

$K_{s}^{i,[1, i-1]}:=\min \left\{k: k M>\sup _{x_{t} \in S_{s, D}^{i}\left(\hat{x}_{t}\right)} W\left(\left(A x_{t}\right)^{i}-\left(A \hat{x}_{t}\right)^{i}\right)\right\}$

This sets the stage for the following result.

Theorem 2.4: Suppose the controllers cooperate while decoding. Let $\Pi_{i}$ be the set of permutations of the sequence $\{1,2, \ldots, n\}$, and $K_{s}^{i,[1, i-1]}$ be as defined above. The following rate is stabilizing under IS D:

$$
R^{*}=\min _{\Pi_{i}} \sum_{i} \log _{2}\left(K_{s}^{i,[1, i-1]}\right)
$$

\section{E. Information Structure E}

Here the sensors do not have access to the actual estimates at the controllers. As in IS D, we need to bound the support size $W\left(\left(A x_{t}\right)^{i}-\right.$ $\left.\left(A \hat{x}_{t}\right)^{i}\right)$, but this time without knowing what the actual value of $\hat{x}_{t}$ is. We thus bound the support size with

$$
\sup _{\hat{x}_{t}}\left\{\max _{x \in V\left(\hat{x}_{t}\right)} a_{i} x-\min _{V\left(\hat{x}_{t}\right)} a_{i} x+D^{i}\right\} .
$$

The controller has to decode the correct bin given only the coset information, as in IS C. Introduce

$$
\begin{aligned}
S_{E}^{i}:= & \left\{x_{t}, \hat{x}_{t}:\left|x_{t}^{j}-\hat{x}_{t}^{j}\right| \leq M, \forall j\right. \\
& \left.W\left(\left(A x_{t}\right)^{k}-\left(A \hat{x}_{t}\right)^{k}\right)<M, \quad k \neq i\right\} .
\end{aligned}
$$

To be able to decode the actual bin uniquely, we need the number of cosets to be equal to at least

$$
K_{-i}^{\prime i}:=\min \left\{k: k M>\sup _{\left\{x_{t}, \hat{x}_{t}\right\} \in S_{E}^{i}} W\left(\left(A x_{t}\right)^{i}-\left(A \hat{x}_{t}\right)^{i}\right)\right\}
$$

which is different from what we had obtained in IS E, due to the presence of the extra $\hat{x}_{t}$ term in the constraint set $S_{E}^{i}$.

Theorem 2.5: There is no difference between the sum rates corresponding to (3) and (4) (corresponding to under IS D and IS E, respectively).

Proof: The objective function is identical in both cases. The only difference is in the constraint set, due to the extra term $\hat{x}_{t}$ in $S_{E}^{i}$.

Suppose there exists a pair $\left\{x_{t}, \hat{x}_{t}\right\}$ in the closure of $S_{E}^{i}$ in (4) that achieves the supremum of $W\left(\left(A x_{t-1}\right)^{i}-\left(A \hat{x}_{t-1}\right)^{i}\right)$. Due to linearity, we have

$$
\left(A x_{t}\right)^{i}-\left(A \hat{x}_{t}\right)^{i}=\left(A\left(x_{t}-\hat{x}_{t}\right)\right)^{i}
$$

Thus, the supremum value is only a function of the difference between the reconstruction value and the actual value. Thus, the value achieved by the pair $\left\{x, \hat{x}_{t}\right\}$ is identical to the one achieved by some arbitrary $\left\{x_{t}-\hat{x}_{t}+\hat{x}_{t}^{\prime}, \hat{x}_{t}^{\prime}\right\}$. Since the reconstruction value is arbitrary, the constraint sets for the evaluation of $K^{i}$ in IS E and IS F are identical. $\diamond$ As in IS D, for a sequential scheme we introduce

$$
\begin{aligned}
S_{E, s}^{i}:= & \left\{x_{t}, \hat{x}_{t}:\left|x_{t}^{j}-\hat{x}_{t}^{j}\right| \leq M, \forall j\right. \\
& \left.W\left(\left(A x_{t}\right)^{k}-\left(A \hat{x}_{t}\right)^{k}\right)<M, k \leq i-1\right\}
\end{aligned}
$$

and

$K_{s}^{\prime i \mid[1, i-1]}:=\min \left\{k: k M>\sup _{\left\{x_{t}, \hat{x}_{t}\right\} \in S_{E, s}^{i}} W\left(\left(A x_{t}\right)^{i}-\left(A \hat{x}_{t}\right)^{i}\right)\right\}$.

We then have the following.

Theorem 2.6: Suppose the controllers cooperate while encoding. Let $\Pi_{i}$ be the set of permutations of the sequence $\{1,2, \ldots, n\}$, and $K^{i,[1, i-1]}$ be as defined above. Then, the following rate is stabilizing:

$$
R^{*}=\min _{\Pi_{i}} \sum_{i} \log _{2}\left(K_{s}^{\prime i \mid[1, i-1]}\right)
$$

\section{F. Information Structure F}

This is the IS where control signals are exchanged with no delay, whereas state estimate transmissions are delayed. In this scheme, state 
information on the stage before the last one is available. Consider the first subsytem. Before quantization

$$
e_{t+1}^{1}=a_{11} e_{t}^{1}+a_{12} \sum_{i} a_{2 i} e_{t-1}^{i}+a_{13} \sum_{i} a_{3 i} e_{t-1}^{i}+\cdots+w_{t}^{1} .
$$

Define $\operatorname{diag}(A)$ as an $n \times n$ diagonal matrix, whose diagonal entries are those of $A$. Let $N:=(A-\operatorname{diag}(A)) A$, and $D$ be an $n \times 1$ vector with entries as the bounds, $D^{i}$, on the noise terms. Now, for the support size $W$ of the estimation errors, we have

$$
W_{t+1} \leq K\left(N^{+}\right) W_{t-1}+K \operatorname{diag}\left(A^{+}\right) W_{t}+K D
$$

Let $\mathcal{K}_{n}$ be the set of diagonal matrices whose non-zero entries have integer reciprocals satisfying the stability condition in system (5). The second-order system in (5) can be reduced to a first-order system in the usual way: Define $W_{t-1}=r_{t}, W_{t}=p_{t}$. Then (5) becomes

$$
\begin{aligned}
{\left[\begin{array}{l}
r_{t+1} \\
p_{t+1}
\end{array}\right] } & =\tilde{B}\left[\begin{array}{l}
r_{t} \\
p_{t}
\end{array}\right]+K\left[\begin{array}{c}
0 \\
w_{t}
\end{array}\right] \\
\tilde{B} & :=\left[\begin{array}{cc}
0 & I \\
K N^{+} & K\left(\operatorname{diag}\left(A_{i i}^{+}\right)\right)
\end{array}\right]
\end{aligned}
$$

and we need $\tilde{B}$ to be stable. This analysis now leads to the following result.

Proposition 2.3: Consider system (1), with information structure $F$. Let $K$ be the solution of the following optimization problem:

$$
\max _{\mathcal{K}_{n}} \operatorname{det}(K)=: \operatorname{det}\left(K^{*}\right)
$$

Then the rate

$$
R^{*}=\sum_{i} \log _{2}\left(\operatorname{det}\left(K^{*}\right)\right)
$$

is stabilizing.

Proposition 2.4: There exists a solution to (6).

Proof: We have $\left|[\tilde{B} x]_{i}\right| \leq\|\tilde{B}\|_{1}\|x\|_{\infty}, \forall i=1, \ldots, 2 n, \forall x$, where $\|\tilde{B}\|_{1}=\max _{i} \sum_{j=1}^{2 n}\left|\tilde{B}_{(i, j)}\right|,[\tilde{B} x]_{i}$ denotes the $i$ 'th element of the vector $\tilde{B} x$, and $\tilde{B}_{(i, j)}$ denotes the $i j$ 'th entry of the matrix $\tilde{B}$. We then have

$$
(\tilde{B} x)^{T} \tilde{B} x \leq 2 n\left(\|x\|_{\infty}\right)^{2}\left(\|\tilde{B}\|_{1}\right)^{2}, \forall x .
$$

Let $\lambda_{m}$ be an eigenvalue of $\tilde{B}$ with maximum absolute value, and $x$ be its corressponding eigenvector. We then have $\left|\lambda_{m}\right|^{2} x^{T} x \leq$ $2 n\|\tilde{B}\|_{1}^{2}\left(\|x\|_{\infty}\right)^{2}$, and since $\left(\|x\|_{\infty}\right)^{2} \leq x^{T} x$, this implies,

$$
\left|\lambda_{m}\right|^{2} \leq 2 n\|\tilde{B}\|_{1}^{2}
$$

Now, let $\left|\lambda_{m}\right| 7>1$. Note that due to the structure of $\tilde{B}$, the corresponding, possibly complex, eigenvector is in the form $\left[y^{T}, \lambda_{m} y^{T}\right]^{T}$. This, in turn, implies that

$$
K N^{+} y+K\left(\operatorname{diag}\left(A_{i i}^{+}\right)\right) \lambda_{m} y=\lambda_{m}^{2} y \text {. }
$$

Defining a new matrix (with possibly complex entries)

$$
S_{\lambda_{m}}:=\left[\left(1 / \lambda_{m}\right) K N^{+}+K\left(\operatorname{diag}\left(A_{i i}^{+}\right)\right)\right]
$$

we have $S_{\lambda_{m}} y=\lambda_{m} y$. But using an argument similar to the one that led to (7), it suffices to bound

$$
\sup _{\lambda_{m}:\left|\lambda_{m}\right| \geq 1} \sqrt{n}\left\|S_{\lambda_{m}}\right\|_{1}
$$

in order to bound the eigenvalues of $S$, which is possible by an appropriate selection of $K$ : Let $k=n C\left(\left\|N^{+}\right\|_{1}+\left\|\left(\operatorname{diag}\left(A_{i i}^{+}\right)\right)\right\|_{1}\right)$, and pick $K=(1 / k) I_{n \times n}$, which would lead to

$$
\sup _{\left|\lambda_{m}\right| \geq 1}\left\|\left(1 / \lambda_{m}\right) K N^{+}+K\left(\operatorname{diag}\left(A_{i i}^{+}\right)\right)\right\|_{1}<1 / n .
$$

Thus, $\left|\lambda_{m}\right|<1$. Now, since there is only a finite number of solutions that are better than the one proposed, the optimization problem (6) has a solution.

Proposition 2.5: If $A$ is diagonal, (6) leads to a sum rate of

$$
R^{*}=\sum_{i} \log _{2}\left(C\left(\left|\lambda_{i}\right|\right)\right)
$$

Proof: Proof follows from the observation that, in the diagonal case, there is no need for the controllers to share their state estimates. $\diamond$

\section{CONCLUDING REMARKS}

This note has studied communication rate requirements for decentralized control systems, with the rates required for centralized schemes being strictly lower. We have shown that the information sharing architecture determines communication requirements and complexity. It is known that in decentralized control systems time-varying policies could lead to better performance than time-invariant policies [6], and hence one would expect a similar type of an improvement in the context of the problem here by resorting to time-varying policies [14]. We have not included here, due to page limitations, any numerical examples illustrating the results. Simulations can be found in [12].

\section{REFERENCES}

[1] M. Santori and K. Zech, "Fieldbus brings protocol to process control," IEEE Spectrum Magazine, vol. 33, pp. 60-64, Mar. 1996.

[2] R. K. Jurgen, "Coming from detroit: Network on wheels," IEEE Spectrum Mag., pp. 53-59, Jun. 1986.

[3] J. A. Fax and R. M. Murray, "Information flow and cooperative control of vehicle formations," IEEE Trans. Autom. Control, vol. 49, pp. 1465-1476, Sept. 2004.

[4] S. Wang and E. J. Davison, "On the stabilization of decentralized control. systems," IEEE Trans. Autom. Control, vol. 18, pp. 473-478, Oct. 1973.

[5] J. P. Corfmat and A. S. Morse, "Decentralized control of linear multivariable systems," Automatica, vol. 12, pp. 479-497, Sept. 1976.

[6] B. D. O. Anderson and J. B. Moore, "Time-varying feedback decentralized control," IEEE Trans. Autom. Control, vol. 26, pp. 1133-1139, Oct. 1981.

[7] S. Yüksel and T. Başar, "Quantization and coding for decentralized LTI systems," in Proc. 42nd IEEE Conf. Dec. Contr., Maui, HI, Dec. 9-12, 2003, pp. 2847-2852.

[8] L. Xiao, M. Johansson, H. Hindi, S. Boyd, and A. J. Goldsmith, "Joint optimization of communication rates and linear systems," IEEE Trans. Autom. Control, pp. 148-153, Jan. 2003. 
[9] A. S. Matveev and A. V. Savkin, "Stabilization of multisensor networked control systems with communication constraints," in Proc. Asian Contr. Conf., Melbourne, Australia, July 2004.

[10] S. Tatikonda, "Some scaling properties of large distributed control systems," in Proc. 42nd IEEE Conf. Dec. Contr., Maui, Hawaii, Dec. 2003 , pp. 3142-3147.

[11] G. N. Nair, R. J. Evans, and P. E. Caines, "Stabilising decentralised linear systems under data rate constraints," in Proc. 43rd IEEE Conf. Dec. Contr., Bahamas, Dec. 2004, pp. 3992-3997.

[12] S. Yüksel, "State Estimation and Control for LTI Systems over Communication Channels," , University of Illinois at Urbana-Champaign, Urbana-Champaign, IL, Oct. 2003, M.S. thesis.

[13] S. Yüksel and T. Başar, "On the absence of rate loss in decentralized sensor and controller structure for asymptotic stability," in Proc. IEEE Amer. Contr. Conf., Minneapolis, MN, Jun. 2007.

[14] S. Yüksel and T. Başar, "Decentralized multi-controller stabilizability over communication channels," in Proc. European Contr.Conf., Kos, Greece, Jun. 2007.

[15] J. D. Slepian and J. K. Wolf, "Noiseless coding of correlated information sources," IEEE Trans. Inf. Theory, vol. 19, pp. 471-480, Jul. 1973.

[16] S. S. Pradhan and K. Ramchandran, "Distributed source coding using syndromes (DISCUS): Design and construction," IEEE Trans. Inf. Theory, vol. 49, pp. 626-643, Mar. 2003.

[17] J. Kusuma, L. Doherty, and K. Ramchandran, "Distributed compression for sensor networks," in Proc. IEEE Conf. Image Processing, Thessaloniki, Greece, Oct. 2001.

[18] A. D. Wyner and J. Ziv, "The rate-distortion function for source coding with side information at the decoder," IEEE Trans. Inf. Theory, vol. 22, no. 1, pp. 1-11, Jan. 1976.

[19] S. Tatikonda, A. Sahai, and S. Mitter, "Stochastic linear control over a communication channel," IEEE Trans. Autom. Control, vol. 49, no. 9, pp. 1549-1561, Sep. 2004.

\section{On the Gap Between Positive Polynomials and SOS of Polynomials}

Graziano Chesi, Senior Member, IEEE

\begin{abstract}
This note investigates the gap existing between positive polynomials and sum of squares (SOS) of polynomials, which affects several analysis and synthesis tools in control systems based on polynomial SOS relaxations, and about which almost nothing is known. In particular, a matrix characterization of the PNS, that is the positive homogeneous forms that are not SOS, is proposed, which allows to show that any PNS is the vertex of an unbounded cone of PNS. Moreover, a complete parametrization of the set of PNS is introduced.
\end{abstract}

Index Terms-Hilbert's 17th problem, linear matrix inequality (LMI), optimization, positive polynomial, sum of squares (SOS).

\section{INTRODUCTION}

Positive polynomials play a key role in the analysis and synthesis of control systems. This is due to the fact that conditions for establishing stability of equilibrium points or computing performance indexes of the system such as the $\mathcal{H}_{\infty}$ norm, can be reformulated in terms of positivity of a Lyapunov function and negativity of its time derivative. These functions are usually polynomials as natural extension of the classic quadratic Lyapunov functions in the attempt of achieving less conservative results. Unfortunately, establishing whether a polynomial is positive or not, is still a difficult problem that cannot be solved systematically because it amounts to solving a nonconvex optimization. In order to deal with this problem, gridding methods have been proposed, for example based on the use of Chebychev points, but their conservativeness and computational burden are generally unacceptable, reason that has motivated the search for alternative approaches.

This search has recently provided the sum of squares (SOS) relaxation (among the first contributions on SOS relaxation, see for example [1]). In this approach, the positivity of a homogeneous form (equivalently of a polynomial) is established by checking if it is a SOS of homogeneous forms, operation which amounts to solving a linear matrix inequality (LMI) feasibility problem, i.e., a convex optimization. Due to the existence of powerful tools for solving LMIs [2], SOS relaxations have quickly become an essential tool in control. In robust control, SOS relaxations have been employed to obtain less conservative conditions than those provided by quadratic Lyapunov functions to assess robust stability of linear systems affected by parametric uncertainty, in both cases of time-varying uncertainty [3]-[6] and time-invariant uncertainty [7]-[9]. An analogous use of SOS has been made in the computation of robust performance indexes [10], [11]. SOS have been exploited also in the field of nonlinear systems [12]-[15], hybrid systems [16], [17] and time-delay systems [18]. See also [19]-[22] for further applications of SOS.

"Can any positive homogeneous form be written as a SOS?" This question was made by Hilbert in his 17 th problem and has a negative answer as it is known. It is hence known that, in spite of their popularity, SOS relaxations can be conservative. However, almost nothing

Manuscript received February 9, 2005; revised December 1, 2006. Recommended by Associate Editor M. Fujita.

The author is with the Department of Electrical and Electronic Engineering, University of Hong Kong, Pokfulam Road, Hong Kong (e-mail chesi@eee.hku. hk).

Digital Object Identifier 10.1109/TAC.2007.899083 